\title{
Erratum to: Control over the Preferred Orientation of CIGS Films Deposited by Magnetron Sputtering Using a Wetting Layer
}

\author{
Authors: Yong Yan,* Fan Jiang, Lian Liu, Zhou Yu,* Yong Zhang, and Yong Zhao \\ DOI: $10.1007 / \mathrm{s} 13391-015-5263-\mathrm{y}$
}

On page 59, Zhou Yu is also contributed as a corresponding author and his affiliation is ${ }^{1}$ School of Electrical Engineering, Superconductivity and New Energy R\&D Center (SNERDC), Key Laboratory of Advanced Technology of Materials, Ministry of Education of China, Southwest Jiaotong University, Mail Stop 165\#, Chengdu, China.

\footnotetext{
*School of Electrical Engineering, Superconductivity and New Energy R\&D Center (SNERDC), Key Laboratory of Advanced Technology of Materials, Ministry of Education of China, Southwest Jiaotong University, Mail Stop 165\#, Chengdu 610031, China *Corresponding author: solarcells@126.com

*Corresponding author: yuzhou@home.swjtu.edu.cn
} 